\title{
AVALIAÇÃO DO ENSINO DA DISCIPLINA ENFERMAGEM GERONTOGERIÁTRICA DO CURSO DE GRADUAÇÃO EM ENFERMAGEM DA FURG*
}

\author{
Bárbara Tarouco da Silva', Silvana Sidney Costa Santos²
}

\begin{abstract}
RESUM O: A população idosa brasileira vem crescendo de forma acelerada trazendo a necessidade de capacitação dos profissionais da saúde, princi pal mente dos enfermei ros. O bjetivou-se aval iar o ensino da disciplina Enfermagem Gerontogeriátrica. Pesquisa qual itativa do tipo estudo de caso, real izado no Departamento de Enfermagem da Fundação U niversidade F ederal do Rio G rande/RS (FURG ). Na col eta dos dados usou-se a entrevista gravada com o total de 26 alunos. A través da análise temática surgiram as seguintes categorias: Conteúdos ministrados; M udança de atitude quanto ao idoso após a realização da disciplina; Condições adquiridas ou não adquiridas no cuidado do idoso após a disciplina; Sugestões. A pesquisa mostrou-se uma ferramenta de avaliação para a disciplina Enfermagem Gerontogeriátrica que foi inserida como disciplina obrigatória no Projeto Político Pedagógico (PPP) do Departamento de Enfermagem da FURG, considerando-se que o objeto da Enfermagem é o ser humano, sendo este: criança, adolescente, adulto ou idoso.
\end{abstract}

DESCRITORES: Enfermagem; Ensino; Idoso; Formação profissional.

\section{TEACHING EVALUATION OF THE GERONTOGERIATRIC NURSING DISCIPLINE IN THE NURSING GRADUATION COURSE AT FURG}

ABSTRACT: Brazilian aged population has been increasing fast, bringing about the need for health professionals' qualification, specially nurses. This study objectified the teaching evaluation of the Gerontogeriatric nursing discipline. Q ualitative research, casestudy type, performed by the Nursing D epartment of Fundação Universidade F ederal do Rio Grande (FURG). During data collection, recorded interviews were used with a total of 26 students. Through the thematic analysis, the following categories emerged: I Teaching Contents; II - A ttitude change to aged people after the classes; III - Conditions acquired or not in elders' care after the discipline; IV - Suggestions. The research revealed as an evaluation tool for the Gerontogeriatric nursing discipline, included as obligatory in the Pedagogical Political Project of the FURG 's N ursing Department, considering that the nursing object is the human being: child, adolescent, adult or aged.

DESCRIPTORS: Nursing; Education; A ged; Professional formation.

\section{EVALUACIÓN DE LA ENSEÑANZA DE LA DISCIPLINA ENFERMERÍA GERONTOGERIÁTRICA DEL CURSO DE GRADUACIÓN EN ENFERMERÍA DE LA FURG}

RESUM EN: La población anciana brasileña viene creciendo de forma acelerada, trayendo la necesidad de la calificación de los profesionales de la salud, principalmente de las enfermeras. Este estudio tiene como objetivo evaluar la enseñanza de la disciplina Enfermería Gerontogeriátrica. Investigación cualitativa del tipo estudio de caso, realizada en el departamento de enfermería de la Fundación U niversidad de Rio Grande/RS (FURG). En la colección de los datos se fue utilizada entrevista grabada con el total de 26 alumnos. A través del análisis temático surgieron las siguientes categorías: Contenido dado; Cambio de la actitud cuánto al anciano después de la realización de la disciplina; Las condiciones adquiridas o no adquiridas en el cuidado de las pernonas mayores después de la disciplina; Sugerencias. La investigación reveló una herramienta de la evaluación para la disci plina de Enfermería Gerontogeriátrica que fue insertada como disciplina obligatoria en el Proyecto Político Pedagógico (PPP) del departamento de enfermaría de la FU RG, en vista de que el objeto del oficio de la enfermera es el ser humano, siendo éste: niño, adolescente, adulto 0 anciano.

DESCRIPTORES: Enfermería; Educación; A nciano; Formación profesional.

\footnotetext{
* Trabal ho financiado pelo PROBIC/FURG/CNPq

${ }^{1}$ A luna do Curso de Graduação em Enfermagem da Fundação U niversidade Federal do Rio Grande - FU RG. Integrante do Grupo de Estudo e Pesquisa em Enfermagem Gerontogeriátrica - GEP-GERON/FURG/CNPq.

${ }^{2}$ Enfermeira. D outora em Enfermagem. Especialista em Gerontologia - SBGG. Professora do Departamento de Enfermagem da FURG. Líder do GEPGERON/FURG/CNPq.
} 


\section{INTRODUÇÃO}

A A ssociação Brasileira de Enfermagem $(A B E n)$, após realizar várias oficinas e seminários estaduais, regionais e nacional e também após um longo período de processo de ação e reflexão vol tado à formulação dos currículos, apresentou e discutiu de forma participativa uma proposta curricular que foi encaminhada ao M inistério da Educação e Cultura (MEC), sendo aprovada com algumas alterações através da Portaria $n .01 .721$, de 15 de dezembro de 1994. 0 currículo de graduação de Enfermagem foi apresentado em seis áreas assim distribuídas: fundamentação básica de Enfermagem, métodos e técnicas na Enfermagem; E nfermagem na assistência à formação enascimento do ser humano; Enfermagem na assistência à saúde da criança e do adolescente; Enfermagem na assistência ao adulto e ao idoso. Ressaltamos que quanto ao idoso foi registrado: "pretende-se que 0 aluno adquira conhecimentos científicos necessários ao desenvolvimento da assistência integral ao ser humano adulto e idoso nos diversos níveis de atenção"; Enfermagem e administração(1:9).

Com a aprovação, pelo MEC, da Portaria n.1.721/94, algumas modificações foram realizadas, apresentando as matérias: a) Bases Biológicas e Sociais da Enfermagem; b) Fundamentos de Enfermagem; c) A ssistência de Enfermagem e d) A dministração em Enfermagem. Nesta proposta 0 termo idoso foi excluído, tirando das escolas de Enfermagem o compromisso da inserção de conteúdos relativos ao cuidado ao ser humano com 60 anos e mais. Alguns enfermeiros, de forma equivocada, entenderam que o processo de envelhecimento, o idoso ea vel hice estariam inseridos no estudo sobre 0 adulto, não diferenciando estas populações, desconsiderando suas especificidades ${ }^{(1: 9)}$.

Outra preocupação quanto ao ensino sobre 0 idoso, o envel hecimento e a vel hice surgiu através da Política Nacional do Idoso, instituída pela Lei $n$. 8.842 de 04 de janeiro de 1994, a qual normaliza esta Política e traz, na área de educação, a possibilidade de inserção de conteúdos sobre o envel hecimento, nos currículos mínimos e nos diversos níveis de ensino, eliminando preconceitos e produzindo novos conhecimentos sobre este tema; inclusão da geriatria e da gerontologia nos currículos dos cursos superiores; elaboração de programas educativos, principalmente nos meios de comunicação, com a finalidade de informar a população sobre o processo de envelhecimento; estímulo a programas que adotem modalidades de ensino à distância, adequados às condições do idoso(2).

Com 0 aumento da população idosa surge a necessidade de capacitar recursos humanos em Enfermagem para atender a demanda de idosos que procuram os serviços de saúde. A população de idosos riograndinos no ano de 2000 era de 20.794 pessoas, ou seja, pouco menos de $10 \%$ do total da população. Q uanto ao atendimento hospitalar, no período de 2000 a 2004 houve 7.192 internações de pessoas com mais de 60 anos no Hospital Universitário Miguel Riet Correa únior ${ }^{(3)}$. Esses dados reforçam a importância da formação de profissionais capacitados a cuidarem dessa parte da população.

Em 1996, a regulamentação da $L$ ei de Diretrizes e Bases da Educação (LDB), n. 9.394/96, em seu artigo 53, atribui ao MEC a competência de fixar os currículos dos seus cursos e programas, através das Comissões de Especialistas do Ensino de Graduação, estabelecendo as Diretrizes Curriculares Nacionais. Desta forma, a Comissão de Especialistas de Enfermagem (CEEn), inserida na Secretaria do Ensino Superior (SESU), do Departamento de Política de Ensino Superior (DEPES), no MEC, elaborou um modelo de enquadramento das Propostas de Diretrizes Curriculares, que caminhou em descompasso com as discussões real izadas nas organizações de ensino, nas entidades de classe e defendidas pela A BE $n^{(4)}$.

No final da década de 90 , com a mudança na equipe que integra a Comissão de Especialistas de Ensino de Enfermagem do MEC, surgiu uma outra proposta curricular para os cursos de graduação em Enfermagem, que corroborou os princípios curriculares elaborados pela $A B E n$, inclusive contemplando o ser humano idoso na área da assistência de Enfermagem. Essa proposta está direcionada para a elaboração de um Projeto Político Pedagógico (PPP) que contemple as dimensões necessárias à formação do enfermeiro, tornando-se, após modificações estabelecidas pelo Conselho Nacional de E ducação - Câmara de E ducação Superior, a Resolução CNE/CES n.3, de 7 denovembro de2001, sobre as Diretrizes Curriculares Nacionais do Curso de Graduação em Enfermagem ${ }^{(5)}$.

Tornou-se imprescindível para o Brasil um investimento efetivo em programas de suporte para idosos como aposentadorias e pensões adequadas, oferta de serviços alternativos como o Centro-Dia, 0 
Hospital-Dia, apoios em áreas como alimentação, transporte, assistência médica voltada aos problemas dos idosos, serviços de orientação e atividades culturais e outros. Também era necessário para os profissionais um investimento em salários justos, condições adequadas de trabal ho, cursos e capacitação nesta área específica, implantação e implementação de ações interdisci plinares e transdisciplinares, dentre outros ${ }^{(5)}$.

Porém, o mais importanteé adequar os serviços de saúde e, principalmente, formar os profissionais, para bem cuidar do ser humano idoso, o que se apresenta um desafio, considerando-se a (in)visibilidade desta temática nos currículos dos cursos de graduação da Enfermagem brasileira no período estudado.

0 trabalho teve como objetivo realizar um estudo de caso $\mathrm{H}$ istórico-organizacional da disciplina Enfermagem Gerontogeriátrica ministrada no Departamento de Enfermagem da Fundação U niversidade Federal do Rio Grande (FURG), a partir da opinião dos alunos que a cursaram.

\section{DESCRIÇÃO DO MÉTODO}

Pesquisa com abordagem qualitativa do tipo estudo de caso. Existem alguns tipos de estudo de casos e entre eles destacamos o Estudo de Caso Histórico-organizacional e neste 0 interesse do pesquisador recai sobre a vida de uma instituição. A unidade pode ser uma escola, uma universidade, uma disciplina, etc. 0 pesquisador deve partir do conhecimento que existe sobre a organização que deseja examinar ${ }^{(6)}$. 0 objeto do nosso estudo é a disciplina Enfermagem G erontogeriátrica.

Os dados foram coletados após aprovação do Comitê de Ética em Pesquisa, tendo sido aprovado com o número de protocolo 23116.002665/2005-79. Os preceitos éticos foram respeitados. Utilizamos um roteiro de entrevista, no qual faziam parte itens relativos à caracterização do aluno e questões rel acionadas à temática do estudo. Também utilizamos a análise de documento para obter informações sobre o Curso de Enfermagem da FURG, por meio do site da Fundação Universidade Federal do Rio Grande (www.furg.br) e informações sobre a disciplina Enfermagem Gerontogeriátrica.

0 estudo teve como sujeitos 26 alunos que cursaram a referida disciplina, no ano de 2005 , os quais concordaram em participar do estudo e assinaram 0
Termo de Consentimento Livre e Esclarecido, elaborado a partir a R esolução 196/96 ${ }^{(7)}$, que normatiza a pesquisa em seres humanos no país.

A análise e interpretação dos dados foi realizada por meio da análise de conteúdo, observando-se a técnica da análise temática, visando descobrir os núcleos de sentido das entrevistas. Esse referencial metodológico levou em consideração o significado dos conteúdos latentes e manifestos, que foram expressos pelos participantes e que certamente revelou outras realidades acerca do ensino tendo como enfoque 0 cuidado ao idoso, na disciplina Enfermagem Gerontogeriátrica, no Departamento de Enfermagem da FURG, direcionando a realização de uma avaliação mais sistemática da disci plina e uma melhor adequação ao atendimento das necessidades do futuro enfermeiro, quanto ao cuidado a pessoas com mais de 60 anos.

\section{RESULTADOS}

Os dados apresentados e discutidos inicialmente caracterizarão a disciplina Enfermagem Gerontogeriátrica, enfocando a Universidade e 0 Departamento de Enfermagem. A pós será caracterizada a população do estudo quanto à distribuição dos sujeitos em relação ao sexo, idade, semestre em que cursou a disciplina. Em seguida apresentamos as questões relacionadas à temática do objeto do estudo, como: conteúdos ministrados, a contribuição da disciplina, as dificuldades encontradas pelos alunos e sugestões para melhor andamento da mesma.

\section{Disciplina Enfermagem Gerontogeriátrica}

0 ensino da disciplina optativa começou no $1^{\circ}$ semestre de 2005, ministrada no Departamento de Enfermagem da Fundação U niversidade do Rio G rande (FURG), tendo uma carga horária de 60 horas e teve como ementa o Processo de envelhecimento, a Política Nacional do Idoso, a Promoção da saúde do idoso e a especificidade do cuidado de Enfermagem Gerontogeriátrica.

A Fundação Universidade Federal do Rio $G$ rande é uma instituição de ensino superior que surgiu de esforços col etivos eindividuais ${ }^{(8)}$. A FURG enfatiza uma formação geral que contemple tanto a técnica como as humanidades, e que seja capaz de despertar criatividade e espírito crítico. Tem por missão servir com qualidades, orientada por princípios éticos e 
democráticos, de manei ra que o resul tado de sua ação educativa tenha impacto na comunidade, contribuindo para uma melhor qualidade de vida das pessoas e também para o desenvolvimento regional ${ }^{(9)}$.

0 Departamento de Enfermagem da FUR G foi criado em fevereiro de 1997, possibilitando o agrupamento de docentes enfermei ros que, até então, se encontravam dispersos nos departamentos de Ciências E conômicas, A dministrativas e Contábeis, Cirurgia, M aterno-Infantil e M edicina Interna. 0 Departamento surgiu a partir de um processo encaminhado por vinte e três docentes-enfermeiros ao Chefe do Departamento de M edicina Interna em 24 de novembro de $1992^{(10)}$.

0 Curso de Enfermagem da FURG tem como objetivo proporcionar condições para um aprendizado técnico, científico, político, humanístico e ético, favorecendo 0 desenvolvimento de competências e habilidades específicas de um perfil profissional, 0 qual habilite o (a) enfermeiro (a) da FURG a utilizar suas potencial idades como enfermeiro (a) generalista, buscando a resolução de problemas pertinentes à Enfermagem, desempenhando funções assistenciais, administrativas e educacionais $s^{(9)}$.

Os objetivos da disciplina Enfermagem Gerontogeriátrica são: compreender o processo de envelhecimento, percebendo a importância da transição demográfica e epidemiológica e à luz das políticas públicas direcionadas ao idoso; distinguir 0 idoso doente do idoso saudável, estimulando a promoção deste contingente populacional por meio da participação deles em atividades específicas e realizar o cuidado ao idoso e sua família considerando a hospitalização, institucionalização e situação domiciliar. Como metodologia temos as atividades didáticas desenvolvidas em forma de aula expositiva dial ogada, seminários, atividades práticas orientadas, dinâmica de grupo, busca bibliográfica, entre outras. Ressaltam-se as iniciativas de introdução de metodologias de ensino que proporcionem a participação ativa na construção do conhecimento, bem como, o oferecimento dos conteúdos.

$\mathrm{Na}$ disciplina foi proposta a avaliação somativa das atividades teóricas e práticas durante seu desenvolvimento e após a conclusão dos conteúdos. Os conteúdos eram trabalhados com os al unos através da exposição dialogada, dinâmicas de grupo e uso de retro-projetor e multimídia. Depois de terminado o conteúdo de cada aula, os alunos realizavam exercícios propostos pelos professores, aos quais eram avaliados numericamente. No caso, de um aluno faltar, era fornecida a oportunidade de recuperar os pontos perdidos, através da realização de um trabalho.

As atividades práticas foram realizadas numa instituição de longa permanência (ILP), da cidade de Rio Grande e constituíram-se de três visitas ao local. Essas atividades possibilitaram aos alunos conhecer um pouco da realidade dos idosos asilados. Cada aluno aplicou um instrumento de avaliação multidimensional em um idoso. Este instrumento continha questões de identificação, sociais, avaliação cognitiva, funcional e exame físico.

No ano de 2006 houve várias oficinas voltadas à reel aboração do Projeto Pedagógico do Curso (PPC) culminando com a nova proposta curricular que deu início em 2007, já com a inclusão da disciplina Enfermagem Gerontogeriátrica como obrigatória no sexto período, seguindo a estruturação vigente.

\section{Características do grupo investigado}

Participaram deste estudo 26 alunos, 23 do sexo feminino e três do sexo masculino. Evidencia-se que existe uma feminização na Enfermagem, o que pode ser confirmado pelo número de alunas, que é mais significativo do que o de alunos participantes dessa pesquisa.

Q uanto à idade, encontramos al unos entre $20 \mathrm{e}$ 27 anos, tendo como média de idade 22 anos. Dos alunos investigados, doze cursaram a disciplina quando estavam matriculados no 50 semestre; treze cursaram a disciplina quando estavam no $7^{\circ}$ semestre e um aluno cursou quando estava no 8-semestre. Onze dos alunos entrevistados fizeram parte da primeira turma da disciplina Enfermagem Gerontogeriátrica, ou seja, $1^{\circ}$ semestre de 2005 e quinze alunos fizeram parte da segunda turma no $2^{\circ}$ semestre de 2005. Considerando que a disciplina é optativa e que são of erecidas 15 vagas por semestre, portanto, têm-se um número significativo de alunos que procuram esta disciplina.

\section{Categorias identificadas}

\section{Conteúdos ministrados}

Deram uma introdução, [...] sobre a realidade do idoso no Brasil, qual o papel que o governo está desempenhando para ajudá-Ios (suj.2). 
Foi possível conhecer sobre os cuidados e especificidades do idoso (suj.15).

De acordo com as falas foi possível perceber que a disciplina possibilitou aos al unos conhecimento sobre o processo de envelhecimento, sobre as especificidades do ser humano idoso e os cuidados voltados para esta população.

Considerando que a Enfermagem é uma profissão que tem como objetivo cuidar do ser humano saudável ou doente, seja indivíduo, família ou grupo e independente da idade em que este esteja inserido, torna-se responsabilidade dos órgãos formadores introduzirem em seus currículos conteúdos inseridos em disciplinas, ou disciplinas específicas (optativas ou obrigatórias) sobre questões relacionadas ao envelhecimento, ao idoso e à vel hice ${ }^{(5)}$.

A Enfermagem constitui-se em um espaço para estudo, inclusão e discussão sobre o cuidado humano ${ }^{(11)}$. Cuidado este voltado para todas as fases da vida do ser humano, ou seja, criança, adolescente, adulto e idoso, levando em consideração as peculiaridades de cada um.

\section{Conteúdos ministrados com algumas ressalvas}

Faltou mais abordagem sobre as patologias dos idosos, mais cuidados de enfermagem voltados aos idosos [...] talvez deveria ter sido abordado fundamentos específicos para os idosos (suj. 1).

A ementa desta disciplina diz respeito à promoção da saúde do idoso e as questões legislativas, além de três doenças que foram abordadas na primeira turma (osteoporose, depressão e doença de Alzheimer). 0 conteúdo de osteoporose foi excluído do programa por solicitação dos alunos da primeira turma e comprometimento da professora de Saúde do A dulto, incluí-lo em seus conteúdos.

\section{Mudança de atitude em relação ao idoso}

Não o vejo mais como um adul to e sim, faço diferença pelas suas alterações físicas e emocionais devido à idade (suj.13).

Você começa a mudar algumas condutas, sabe de que forma agir, começa a saber de algumas necessidades. M as de certa forma eu mudei sim [...] (suj.11).
Verificamos que os al unos compreenderam que o ser humano idoso não deve ser visto como um adulto, e sim como um ser dotado de especificidades, al guns com problemas físicos, psicológicos, sociais e principalmente com limitações que ocorrem ao longo dos anos. Os idosos apresentam necessidades específicas e por isso se faz necessário a formação de recursos voltados a atender essas necessidades.

$\mathrm{H}$ abilidades adquiridas ou não adquiridas no cuidado do idoso

Contribuiu para melhor cuidarmos dos idosos, termos melhor visão/atuação nos cuidados e atividades realizadas [...]. Sinto-me mais instrumentalizada, com mais conhecimentos passíveis de prática, para 0 cuidado em saúde do idoso (suj. 23).

De acordo com o depoimento, verificamos que os al unos adqui riram segurança e preparo para realizar o cuidado destinado ao idoso, através do conhecimento obtido durante as aulas, possibilitando uma melhor instrumentalização do futuro profissional.

Adquiri conhecimentos e estes me possibilitaram assistir melhor 0 idoso em todas as suas necessidades (suj. 24).

Percebemos que foram adquiridas condições, as quais proporcionaram uma assistência mais qualificada ao idoso, devido o conhecimento das necessidades e dificuldades que este ser apresenta.

Eu acho que acrescentou sim, porque me deu um pouco mais de conhecimento sobre as transformações, por exemplo, hoje eu sei lidar com a perda de memória dos meus avós [...] (suj.11).

De acordo com o conteúdo do relato anterior, a disciplina contribuiu tanto para a formação profissional, quanto para a pessoal, pois acrescentou um maior conhecimento sobre as transformações ocorridas durante o processo de envelhecimento. Também possibilitou uma oportunidade de discussão acerca das questões que envolvem envelhecimento, idoso e velhice.

\section{Dificuldades encontradas}

No final do semestre as últimas aulas foram 
extremamente corridas (suj.9).

Este depoimento foi de um aluno que cursou a primeira turma e esta questão foi corrigida já na segunda turma, partindo da avaliação da primeira. A Iguns assuntos foram retirados, como Osteoporose, considerando que uma das docentes envolvidas na disciplina Enfermagem Gerontogeriátrica é também professora da disciplina que aborda os agravos clínicos mais freqüentes no adulto e assumiu este conteúdo. Outros assuntos foram condensados.

A gente podia [...] entrar mais no ser idoso quanto aos seus problemas de identificação social [...] antes de começar o conteúdo propriamente dito, porque isso aí vai nos dar pistas [...] sobre quem é o idoso e a partir disso a gente consegue conhecer por que se processam determinadas coisas, por que ele [o idoso] é excluído socialmente (suj.11).

Percebemos que por mais que convivamos com pessoas idosas, como referiram alguns alunos, ainda é difícil lidar com eles e entendê-los, uma vez que, como qualquer ser humano, apresentam suas especificidades e dificuldades. M uitas são as limitações e adequações que surgem ao longo de suas vidas, como perda do trabalho, por meio da aposentadoria; perda de cônjuges e amigos, que predispõem à ocorrência de problemas de ordem emocional, que precisam ser compreendidos pelos que Ihes rodeiam.

A velhice é acompanhada por mudanças de ordem física, psicológica e social, podendo ser uma etapa difícil quando as pessoas não conseguem adaptarsea essas mudanças. A angústia, as perdas, as limitações físicas, a proximidade da morte e a ausência de perspectiva são dificul dades encontradas nesta fase. A sociedade tem papel determinante nessas mudanças que acompanham o envel hecimento, influenciando positivo ou negativamente nas condições da vida dos que estão envelhecendo(12).

\section{Sugestões}

Falta muito o direcionamento patológico, de repente a área hospitalar (suj.3).

Esses aspectos já estão contemplados em outras disciplinas e estágios, como foi mencionado anteriormente. Quanto às doenças, a maioria é vista em outras disciplinas do curso. Percebemos nestas falas a necessidade de alguns alunos quanto ao aprendizado sobre doenças, quando na disciplina Enfermagem Gerontogeriátrica o enfoque se dá na promoção da saúde. Caberá às docentes da disciplina, ao apresentar o programa/cronograma deixarem claro, aos alunos, a finalidade maior da disciplina que é de direcionar para o enfoque de promoção da saúde dos idosos.

\section{CONSIDERAÇÕES FINAIS}

Com o aumento da população idosa nos últimos anos torna-se necessária a inclusão de disciplinas voltadas à capacitação de recursos humanos nos currículos dos cursos da área da saúde, permitindo assistir às necessidades básicas dessa população, já que há um aumento da procura pelos serviços de saúde.

Este trabalho objetivou um estudo de caso Histórico-organizacional do ensino da disciplina Enfermagem Gerontogeriátrica do Departamento de Enfermagem da FURG, através de entrevistas realizadas com os alunos que cursaram a referida disciplina no $1^{0}$ e 20 semestre de 2005. A partir das respostas obtidas foram realizadas mudanças, adaptando a disciplina à formação do al uno da FU RG, contribuindo na aquisição de habilidades, competências e na habilitação para o cuidado, possibilitando a oportunidade de enfrentar as demandas de idosos nos serviços de saúde.

A pós reestruturação curricular no Departamento de Enfermagem da FURG a disciplina Enfermagem Gerontogeriátrica foi introduzida como disciplina obrigatória, a partir da demanda dos al unos e principalmente, pela necessidade de instrumentar a (o) futura (o) enfermeira (o) no cuidado ao ser humano: criança, adulto e idoso.

\section{REFERÊNCIAS}

1. M inistério de E ducação e Cultura (BR ). Comissão de Especialistas de Enfermagem. Proposta preliminar de currículo mínimo para os cursos de graduação em enfermagem. B rasília: 1989. 12 f. (M imeografado).

2. Ministério da Previdência e A ssistência Social (BR). Secretaria de Assistência Social. Lei n. 0 8.842/94 e Decreto n. ํ 1.948/96. B rasília:1997.

3. Tier CG et. al. Política de Saúde do Idoso: iniciativas identificadas no município de Rio G rande-RS. Cogitare Enferm 2006; 11(1): 39-43.

4. Vale EG, Guedes M V C. A nova política de educação e 
suas implicações nos cursos de graduação de enfermagem - apreciação crítica da A ssociação B rasileira de Enfermagem - ABEN. In: A nais do 51으 Congresso Brasileiro de Enfermagem.Florianópolis: ABEn; 1999. p. 370-8

5. Santos SSC. 0 ensino da enfermagem gerontogeriátrica no Brasil de 1991 a 2000 à luz da complexidade de Edgar M orin. Florianópolis: UFSC; 2003.

6. Chizzotti A. Pesquisa em ciências humanas e sociais. São Paulo: Cortez; 1995.

7. M inistério da Saúde (B R). Consel ho N acional de Saúde. Resolução n. 196, de 10 de outubro de 1996. Dispõe sobre diretrizes e normas regulares de pesquisa envolvendo seres humanos. B rasília: 1996.

8. Alves FN. FURG: 35 anos a serviço da comunidade. Rio Grande: FURG; 2004.

9. Fundação Universidade do Rio Grande (FURG). Departamento de Enfermagem. Projeto Político Pedagógico do Curso de Enfermagem. Rio Grande: FURG ; 2005.

10. M eirelles A LB. Ciências da Saúde. In: A lves F das N, organizador. Fundação Universidade Federal do Rio Grande: 35 anos a serviço da comunidade. Rio Grande: FURG ; 2004.

11. Waldow VR. Cuidado humano: o resgate necessário. Porto A legre: Sagra L uzzatto; 1998.

12. Teixeira MH. A spectos psicológicos da velhice. In: Caldas CP, organizador. A saúde do idoso: a arte de cuidar. Rio de J aneiro: UERJ; 1998. 